\title{
PROPOSTA PARA A CONSTRUÇÃO UM SISTEMA INFORMATIZADO PARA GESTÃO INTEGRAL DE RISCOS DE DESASTRES NATURAIS (SIGRID) NO CENÁRIO BRASILEIRO
}

\author{
Leandro Torres Di Gregorio ${ }^{1}$ \\ Carlos Alberto Pereira Soares ${ }^{2}$ \\ Silvia Midori Saito ${ }^{3}$ \\ Erico Soriano ${ }^{4}$ \\ Luciana de Resende Londe ${ }^{5}$ \\ Marcos Pellegrini Coutinho ${ }^{6}$
}

\begin{abstract}
Resumo: Partindo-se de uma pesquisa bibliográfica nos múltiplos assuntos relacionados à gestão integral de riscos de desastres e da observação participante dos autores por meio de vivência em situações operacionais e interinstitucionais no cenário de proteção e defesa civil brasileiro, propôs-se a criação de um Sistema Informatizado para Gestão Integral de Riscos de Desastres Naturais (SIGRID). A proposta foi construída a partir de uma análise conjugada dos principais sistemas nacionais em voga (Sistema de Monitoramento e Alertas de Desastres Naturais/MCTI, Sistema de Monitoramento da Ocupação Urbana/MCid e Sistema de Informações Sobre Desastres/MI) que, além de serem complementares, também teriam a função de congregar informações e sistemas já existentes nos âmbitos federal, estadual e municipal, que se mostrem afins com suas respectivas missões. Neste contexto, foram analisadas as necessidades e o arranjo interoperacional conceitual entre os sistemas existentes, bem como foram definidas as principais características e funcionalidades de um sistema informatizado cuja missão seja conectar as atividades relacionadas à Gestão Integral de Riscos de Desastres no Brasil, levando em conta as peculiaridades do pacto federativo do país. A proposta deste sistema está alinhada com a Lei 12.608/12.
\end{abstract}

Palavras-chave: Sistemas de Gestão; Gestão Integral de Riscos; Gestão Pública; Desastres Naturais; Resiliência.

\section{Proposal for an Integrated System for Natural Disasters Risk Management in the Brazilian Scenario}

Abstract: This work presents a design for an Integrated Management System of Natural Disasters Risks (SIGRID), based on literature review about integral management of disasters

\footnotetext{
${ }^{1}$ Graduação em Engenharia Civil pela Universidade Federal do Rio de Janeiro, mestrado e doutorado em Engenharia Civil pela Universidade Federal Fluminense (gestão da recuperação pós-desastre), especialização em Gestão de Emergências e Desastres pela Faculdade Integrada da Grande Fortaleza. E-mail: leandro.torresdigregorio@gmail.com

${ }^{2}$ Doutor em engenharia (pesq. operac. e gerenc. de producao) pela Universidade Federal do Rio De Janeiro. atualmente e professor associado iii da Universidade Federal Fluminense. E-mail: carlos.uff@globo.com

${ }^{3}$ Graduação em Geografia pela Universidade Estadual de Maringá, mestrado e doutorado em Geografia pela Universidade Federal de Santa Catarina. Atualmente é pesquisadora do Centro Nacional de Monitoramento e Alertas de Desastres Naturais (CEMADEN), vinculado ao Ministério de Ciência, Tecnologia e Inovação (MCTI). E-mail: silvia.saito@cemaden.gov.br

${ }^{4}$ Graduação em Geografia (licenciatura e bacharelado) pela Universidade Estadual Paulista Júlio de Mesquita Filho-UNESP, mestrado em Geografia pela Universidade Estadual Paulista Júlio de Mesquita Filho-UNESP, e doutorado em Ciências da Engenharia Ambiental pela Escola de Engenharia de São Carlos da Universidade de São Paulo EESC-USP. Trabalha como Analista de Pesquisa em Desastres Naturais no Centro Nacional de Monitoramento e Alertas de Desastres Naturais (CEMADEN). E-mail: erico.soriano@cemaden.gov.br ${ }^{5}$ Doutorado em Sensoriamento Remoto pelo Instituto Nacional de Pesquisas Espaciais - INPE, mestrado em Engenharia Agrícola (Área de Concentração Água e Solos) pela Universidade Estadual de Campinas - UNICAMP e graduação (Bacharelado e Licenciatura Plena) em Ciências Biológicas pela Universidade Federal de Uberlândia - UFU. E-mail: luciana.londe@cemaden.gov.br

${ }^{6}$ Graduação em Engenharia Florestal pela Universidade Federal de Viçosa e mestrado em Produção Vegetal pela Universidade Estadual do Norte Fluminense Darcy Ribeiro. Atualmente é professor da Universidade Estadual de Goiás. E-mail: marcos.coutinho@cemaden.gov.br DOI: 10.7154/RDG.2013.0026.0005
} 
risks and also on the authors' experience related to operational and inter-institutional situations in the Brazilian protection and defense civil scenario. The combined analysis of the main current Brazilian systems was the starting point for the creation of this proposal. Those systems (System for Monitoring and Warnings of Natural Disasters/MCTI, System for Urban Occupation Monitoring/MCid and System for Information about Disasters/MI) provide complementary information and are responsible for joining information and other related systems in different scales (including municipalities, state and the whole country in some cases). The needs and the conceptual inter-operational arrangement among systems were analyzed and the new system's main characteristics and functions were defined. The proposed system's mission highlights the connection of activities related to the Integral Management of Disasters Risks in Brazil, considering peculiarities of the country's federative pact. The proposal is in accordance with the Brazilian Law 12.608/12.

Key-words: Management Systems; Integral Risk Management; Public Management; Natural Disasters; Resilience.

\section{INTRODUÇÃO}

O desempenho de uma organização está intimamente relacionado ao domínio sobre seus respectivos processos e atividades, seu posicionamento estratégico e relacionamento no ambiente onde atua, à adequação de seus produtos / serviços às necessidades e desejos de seus clientes e demais partes interessadas, ao cumprimento de requisitos normativos e legais, bem como sua capacidade de melhorar seus processos e produtos / serviços de forma contínua e inovadora.

A questão torna-se mais complexa na medida em que o porte da organização aumenta, havendo necessidade de criação de departamentos e mecanismos de controle em diversos níveis, estruturação de processos e métricas de desempenho, bem como a definição de metas a serem atingidas que levem em consideração a interdependência com o restante da organização e com agentes externos. O desafio se intensifica quando se trata de integrar diferentes unidades de negócio de uma mesma empresa, ou ainda, diferentes organizações. Para lidar com esta complexidade, as ferramentas de sistemas de informação mostram-se extremamente úteis. Através do uso destas tecnologias, é possível integrar pessoas e processos, facilitar as comunicações e otimizar o fluxo de trabalho e de informações, estabelecer e aferir o cumprimento de metas, agilizando o ciclo operacional de forma sinérgica e criando uma estrutura de aprendizado (multi)organizacional baseada na melhoria contínua. Quando estas ferramentas de tecnologia da informação são planejadas e utilizadas 
de forma integrada e sistêmica, refletindo a dinâmica de processos da organização, pode-se dizer que se trata de um Sistema Integrado de Gestão.

A dinâmica de interação das atividades direta ou indiretamente relacionadas à proteção e defesa civil também constitui um sistema complexo, tendo como principal característica a interdisciplinaridade e interinstitucionalidade das operações, envolvendo instituições pertencentes a diferentes entes federativos (municípios, estados e união). Uma gestão nesses moldes é um desafio que só poder sem vencido com uma abordagem sistêmica e integrada, facilitada sobremaneira pelo desenvolvimento de um sistema de gestão.

Atualmente no Brasil o que se tem constatado é que diferentes sistemas vêm sendo propostos e/ou construídos por diferentes organizações que fazem parte do Sistema Nacional de Proteção e Defesa Civil (SINPDEC), relacionados às suas respectivas missões institucionais. Como exemplo, citam-se o Sistema de Monitoramento e Alerta de Desastres Naturais, desenvolvido e operado pelo Centro Nacional de Monitoramento e Alertas de Desastres Naturais (Cemaden), o Sistema de Monitoramento da Ocupação Urbana, proposto pelo Ministério das Cidades (MCid), o Sistema Integrado de Informações Sobre Desastres (S2iD), do Ministério da Integração (MI), dentre outras iniciativas para a construção de sistemas em âmbitos estaduais e até mesmo municipais.

É legítimo que cada instituição busque a excelência de suas operações, dentro de suas peculiaridades de missão e cultura organizacional, sendo para isto necessário que os sistemas individuais retratem a dinâmica interna de seus processos. Entretanto, dado o elevado grau de transversalidade da gestão integral de riscos e a necessidade de comunicação entre os atores, é imprescindível que estes sistemas também sejam pensados visando a integração e compartilhamento de dados com outras instituições co-participantes, de forma automatizada ou semi-automatizada. Ou seja, apesar de estes sistemas, até o momento, terem sido pensados no sentido de trabalhar de forma relativamente independente, o momento atual enseja uma concepção integrada, onde se busque a complementaridade e a sinergia de esforços.

No cenário brasileiro atual, portanto, há necessidade de uma plataforma que integre os diferentes sistemas que estão sendo propostos e/ou construídos, com função de atuar como elemento de ligação, acessar dados passíveis de compartilhamento e distribuí-los, dentro de uma estrutura de hardware e software compatível, garantindo assim a interoperabilidade necessária. Além disso, esta plataforma deve contemplar uma lógica que eventualmente preencha "vazios operacionais" deixados pelos sistemas individuais, que evite a 
superposição de funções e atribuições, além de garantir a extração de parâmetros que permitam criar padrões de interpretação (inteligência) e medir o desempenho do sistema como um todo, de forma transparente.

Este trabalho avalia as necessidades e o arranjo interoperacional conceitual entre os sistemas existentes, bem como define as principais as características e funcionalidades de um sistema informatizado cuja missão seja conectar as atividades relacionadas à Gestão Integral de Riscos de Desastres no Brasil, considerando as peculiaridades do pacto federativo do país. A proposta deste sistema está alinhada com a Lei $12.608 / 12$, que prescreve em seu art. 13 que:

"Fica autorizada a criação de sistema de informações de monitoramento de desastres, em ambiente informatizado, que atuará por meio de base de dados compartilhada entre os integrantes do SINPDEC visando ao oferecimento de informações atualizadas para prevenção, mitigação, alerta, resposta e recuperação em situações de desastre em todo o território nacional".

\section{METODOLOGIA}

A metodologia empregada consistiu em pesquisa bibliográfica nos múltiplos assuntos relacionados à gestão integral de riscos de desastres naturais - prevenção, preparação, resposta e reconstrução (CASTRO, 1999) - seguida da observação participante dos autores por meio de vivência em situações operacionais e interinstitucionais do cenário brasileiro de gestão de riscos.

A observação participante foi realizada por meio de atividades de campo, participação em cursos / eventos e em projetos / reuniões de trabalho com importantes atores do cenário de gestão integral de riscos de desastres naturais brasileiro, com destaque para:

\section{$\checkmark \quad$ Atividades de campo / operacionais:}

- Visita aos municípios de Areal e São José do Vale do Rio Preto - RJ, durante as atividades de resposta ao desastre de janeiro de 2011;

- Visita às defesas civis dos municípios de Blumenau, Ilhota, Itajaí e Rio do Sul, localizados no estado de Santa Catarina, em agosto de 2012;

- Visita às defesas civis nos municípios de Petrópolis, Teresópolis e Nova Friburgo, com a participação do Instituto Estadual do Ambiente - INEA e do Departamento de Recursos Minerais do Estado do Rio de Janeiro - DRM, em setembro de 2012; 
- Visita ao Centro Estadual de Administração de Desastres do Rio de Janeiro - Cestad e à Secretaria de Estadual de Defesa Civil - RJ, em setembro de 2012;

- Visita ao Serviço Geológico Brasileiro - CPRM, em setembro de 2012;

- Visita ao Centro de Operações da Prefeitura do Rio de Janeiro, em setembro de 2012;

- Participação das atividades de monitoramento e alertas de desastres naturais, no Centro Nacional de Monitoramento e Alertas de Desastres Naturais - CEMADEN, e sua interface com as atividades do Centro Nacional de Gerenciamento de Riscos de Desastres - CENAD, de dezembro de 2011 até o momento;

\section{Participação em cursos / eventos:}

-Participação no Simpósio Estadual de Políticas para a Previsão de Deslizamentos de Terra e a Prevenção de Desastres, organizado pela Secretaria Estadual de Planejamento e Gestão - RJ, em julho de 2012;

-Participação no evento Understanding Risks Brazil (URBR), organizado pelo Ministério da Integração Nacional em parceria com o Banco Mundial, em novembro de 2012;

-Participação em curso de capacitação sobre a utilização do sistema S2ID, da Secretaria Nacional de Defesa Civil, nos meses de novembro e dezembro de 2012;

\section{$\checkmark \quad$ Participação em projetos / reuniões de trabalho:}

-Troca de conhecimento com profissionais e pesquisadores do Japão durante a construção da proposta do Projeto Fortalecimento da Estratégia Nacional de Gestão Integrada de Riscos de Desastres Naturais, em conjunto com a Agência Internacional de Cooperação do Japão (JICA), a Agência Brasileira de Cooperação (ABC), O Ministério da Ciência, Tecnologia e Inovação (MCTI), o Ministério das Cidades (MCid), o Ministério da Integração Nacional (MI) e o Ministério de Minas e Energia (MME), em agosto e setembro de 2012;

-Participação de reunião de trabalho sobre a IRP (International Recovery Platform), plataforma da ONU de boas práticas para recuperação / reconstrução pós-desastres, na Secretaria Nacional de Acessibilidade e Programas Urbanos do Ministério das Cidades, em outubro de 2012.

Das atividades de campo, foi possível apreender in loco a dinâmica de trabalho e as necessidades diversas de diferentes instituições do SINPDEC, especialmente no campo 
operacional, bem como a distribuição de responsabilidades no âmbito do pacto federativo. $A$ familiarização com o Sistema Nacional de Monitoramento e Alertas de Desastres Naturais também se inclui neste grupo de atividades.

As atividades relacionadas à participação em cursos/ eventos forneceram um importante panorama das práticas e ferramentas atualmente em discussão no Brasil sobre gestão integral de riscos, em especial o Sistema de Monitoramento da Ocupação Urbana e o Sistema Integrado de Informações Sobre Desastres.

Por sua vez, a participação em projetos / reuniões de trabalho proporcionaram uma visão ampla do arranjo interinstitucional atual na esfera federal e suas peculiaridades, contribuindo para que a proposta do sistema fosse compatível e factível do ponto de vista das missões e interesses institucionais.

\section{A Gestão Integral de Riscos de Desastres e o Contexto Brasileiro}

Os efeitos nocivos do desastre são diretamente proporcionais à vulnerabilidade dos elementos em risco em seus diversos aspectos, quais sejam, física, ambiental, econômica, política, organizacional, institucional, educativa e cultural (VARGAS, 2010), havendo necessidade de planejar e implementar medidas encadeadas e sinérgicas, estruturais e não estruturais, que culminem na redução dos riscos de desastres. Segundo este autor, um resultado desejado se alcança mais eficientemente quando as atividades e os recursos relacionados são geridos como um processo, que transforma insumos em produtos, numa abordagem alinhada com as normas NBR ISO 9001:2000 (Gestão da Qualidade) e NBR ISO 31.000:2009 (Gestão de Riscos).

\section{Necessidades e desafios}

Valencio e Valencio (2011) ao mencionarem o Sistema de Proteção e Defesa Civil brasileiro, apontam a necessidade de dominar, numa perspectiva complexa e multiescalar, um conhecimento aprofundado acerca das condições socioambientais produtoras de riscos múltiplos de desastres e uma coordenação intersetorial eficiente, que sejam capazes de promover uma interação adequada com a sociedade civil, visando à resiliência. Considerando-se a gestão de risco no país, observa-se o caráter extremamente recente de uma preocupação maior no tripé: proteção, previsão e prevenção. A gestão de desastres no 
Brasil concentrava os esforços na atuação durante o desastre, e não na prevenção, caracterizando-se por uma gestão de urgência e gestão de crise (ALMEIDA; PASCOALINO, 2009).

Valencio e Valencio (2011) apontam ainda a necessidade institucional de uma ética de accountability, favorecendo a prestação de contas sobre as estratégias que visem reduzir os desastres ou mitigar os danos e prejuízos. Os pontos apresentados por estes autores estão perfeitamente alinhados com as possibilidades oferecidas por sistemas informatizados de gestão.

O sistema proposto contribuirá fortemente para a sinergia intra e interinstitucional das instituições que compõem o SINPDEC, traduzida em termos de efetividade em suas operações, com impactos diretos na redução de danos e prejuízos associados aos desastres, sejam eles humanos, materiais, patrimoniais, sociais, políticos e/ou institucionais. Ao abordar de forma integrada os macroprocessos de Prevenção, Preparação, Resposta e Reconstrução, espera-se que o SIGRID contribua para a melhoria contínua da gestão integral de riscos no Brasil e consequentemente para o desenvolvimento sustentável da sociedade, refletido num progressivo ganho real de qualidade de vida, atividade econômica e estabilidade social, mesmo após desastres (Figura 1).

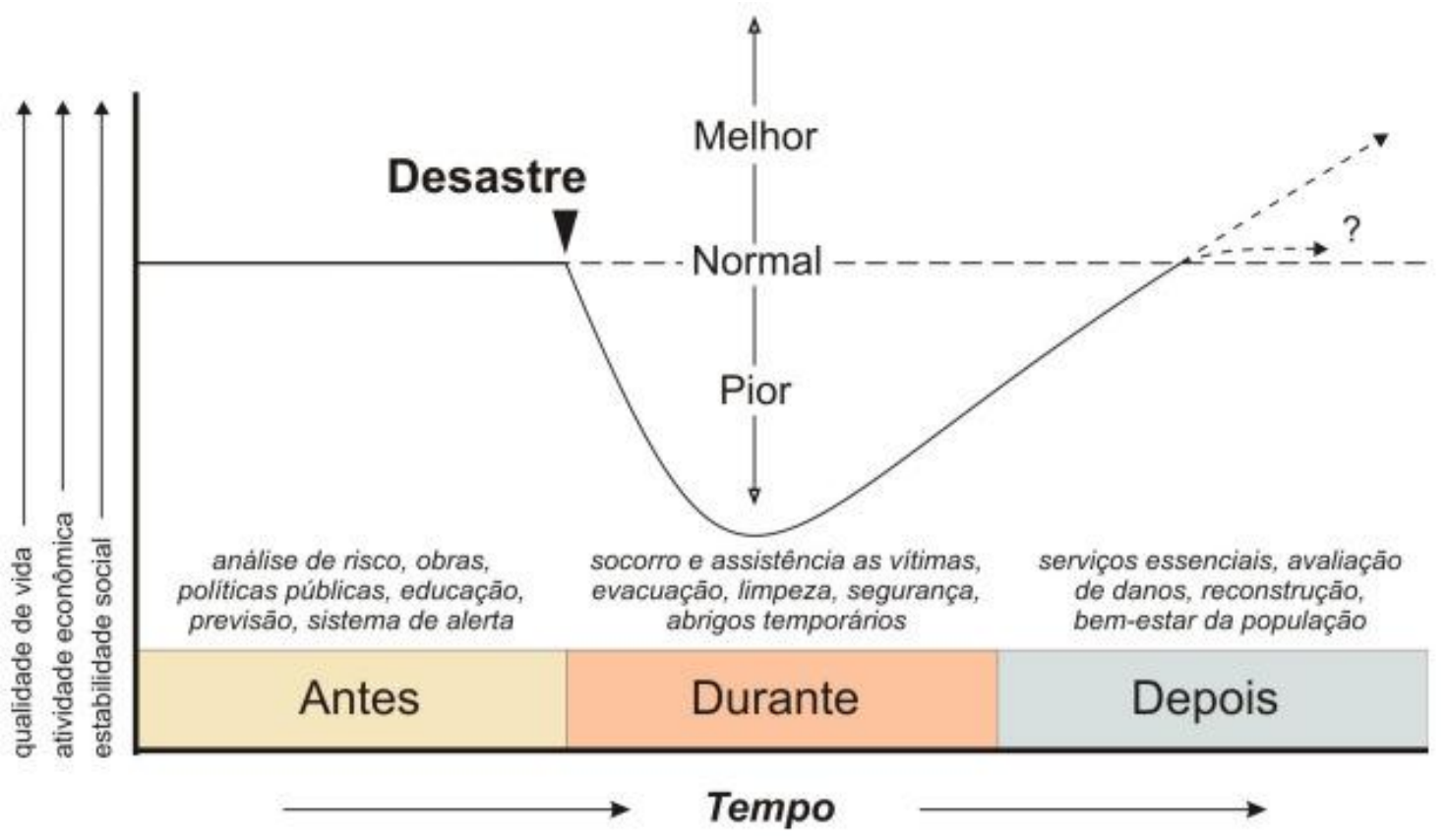

Figura 1: Abordagem de desempenho na gestão integral de riscos. Fonte: MARCELINO (2008).

\section{Abrangência dos macroprocessos}


O Manual de Planejamento em Defesa Civil Volume 1 (CASTRO, 1999), esclarece a abrangência dos macroprocessos de Prevenção, Preparação, Resposta e Reconstrução, que se refletirá na estrutura modular do sistema proposto:

\section{a) Prevenção}

A Prevenção de Desastres compreende a avaliação de riscos de desastres e a redução de riscos de desastres, que por sua vez são desmembradas da seguinte forma:

"A avaliação de riscos de desastres desenvolve-se em três etapas: estudo das ameaças de desastres; estudo do grau de vulnerabilidade dos cenários dos desastres (sistemas receptores e corpos receptivos); sintese conclusiva, objetivando a avaliação e a hierarquização dos riscos de desastres e a definição de áreas de maior risco.

O estudo das áreas de risco permite a elaboração de bancos de dados e de mapas temáticos sobre ameaças, vulnerabilidades e riscos de desastres."

"As ações de redução de riscos de desastres podem ser desenvolvidas com o objetivo de: minimizar a magnitude e a prevalência das ameaças de acidentes ou eventos adversos; minimizar a vulnerabilidade dos cenários $e$ das comunidades em risco aos efeitos desses eventos.

Em ambos os casos, caracterizam-se dois grandes conjuntos de medidas preventivas: medidas não-estruturais, dentre as quais destaca-se o planejamento da ocupação e da utilização do espaço geográfico, em função da definição de áreas de risco, e o aperfeiçoamento da legislação sobre segurança contra desastres; medidas estruturais, também chamadas de medidas de "pedra-e-cal", que têm por finalidade aumentar o nível de segurança intrínseca dos biótopos humanos, através de atividades construtivas" (CASTRO, 1999, p. 18).

\section{b) Preparação}

A Preparação para emergências e desastres tem por objetivo otimizar o funcionamento do SINDEC (Sistema Nacional de Defesa Civil) e, consequentemente, as ações preventivas de resposta aos desastres e de reconstrução, constituindo-se de: desenvolvimento institucional; desenvolvimento de recursos humanos; desenvolvimento científico e tecnológico; mudança cultural; motivação e articulação empresarial; informações e estudos epidemiológicos sobre desastres; monitorização, alerta e alarme; planejamento operacional e de contingência; planejamento de proteção de populações contra riscos de desastres focais; mobilização; aparelhamento e apoio logístico.

\section{c) Resposta}

A Resposta aos desastres compreende as seguintes atividades gerais:

$\checkmark$ Socorro às populações em risco, desenvolvido em três fases: pré-impacto: intervalo de tempo que ocorre entre o prenúncio e o desenvolvimento do desastre; impacto: 
momento em que o evento adverso atua em sua plenitude; limitação de danos: também chamada fase de rescaldo; corresponde à situação imediata ao impacto, quando os efeitos do evento adverso iniciam o processo de atenuação.

$\checkmark$ Assistência às populações afetadas, que depende de atividades: logísticas; assistenciais; de promoção da saúde.

$\checkmark$ Reabilitação dos cenários dos desastres, compreendendo as atividades de: avaliação de danos; vistoria e elaboração de laudos técnicos; desmontagem de estruturas danificadas, desobstrução e remoção de escombros; sepultamento; limpeza, descontaminação, desinfecção e desinfestação do ambiente; reabilitação dos serviços essenciais; recuperação de unidades habitacionais de baixa renda.

\section{d) Reconstrução}

A Reconstrução tem por finalidade restabelecer em sua plenitude: os serviços públicos essenciais; a economia da área; o bem-estar da população; o moral social. Os autores sustentam ainda que, de certa forma, a reconstrução confunde-se com a prevenção e procura: recuperar os ecossistemas; reduzir as vulnerabilidades dos cenários e das comunidades a futuros desastres; racionalizar o uso do solo e do espaço geográfico; relocar populações em áreas de menor risco; modernizar as instalações e reforçar as estruturas e as fundações; recuperar a infraestrutura urbana e rural.

\section{Pacto federativo}

Outra característica importante do contexto brasileiro diz respeito ao pacto federativo. Para representar este modelo, mostra-se adequada uma perspectiva de cone (Figura 2), onde a base contenha os macroprocessos da gestão integral de riscos, e a altura reflita o modelo do pacto federativo, ou seja, a integração necessária entre municípios, estados e união, respeitando-se a autonomia entre os entes. O corpo do cone, portanto, representa a gestão de riscos perpassando todos os entes da federação, em alinhamento com a Lei 12.608 , que estabelece:

\footnotetext{
"Art. 4o São diretrizes da PNPDEC:

I - atuação articulada entre a União, os Estados, o Distrito Federal e os Municípios para redução de desastres e apoio às comunidades atingidas; II - abordagem sistêmica das ações de prevenção, mitigação, preparação, resposta e recuperação".
} 


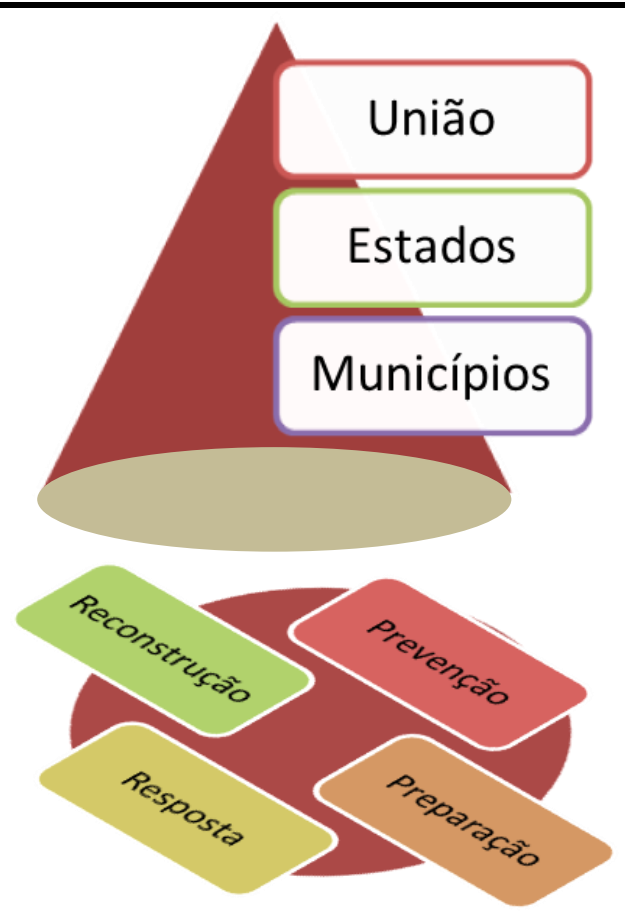

Figura 2: Modelo conceitual cônico da gestão integral de riscos. Fonte: Os autores.

\section{Proposta de Sistema Informatizado para Gestão Integral de Riscos de Desastres}

\section{(Sigrid)}

Para que haja uma abordagem realmente integrada da gestão de riscos no complexo Sistema de Proteção e Defesa Civil brasileiro, há necessidade de uma plataforma de integração de sistemas, dados, informações, comunicações e instituições. Esta ferramenta deve perpassar os municípios, estados e união, ser desenhada a partir de uma visão holística do contexto multi-institucional, permitir identificar e atender as necessidades de forma dinâmica, onde e quando estas se apresentem, e também efetuar as cobranças cabíveis por resultados.

O fluxo de trabalho desta plataforma informatizada, que seria compartilhado por diversas instituições com responsabilidades bem definidas, deve refletir o sequenciamento de processos necessário para uma gestão de riscos efetiva. Ou seja, a operação deve ser participativa, contando com a contribuição de instituições municipais, estaduais e federais cujas atividades possuam interface com a gestão integral de riscos de desastres naturais, no âmbito de suas atribuições e responsabilidades. Para tanto, é necessário que o sistema conte com coordenadores nos níveis federal, estadual e municipal, responsáveis por 
esclarecer, implementar e auxiliar a operação do sistema em seu respectivo nível de atuação. Como não poderia deixar de ser, a coordenação geral de um sistema desta amplitude cabe ao ente federal, porém respeitando-se o pacto federativo.

Importa ressaltar que o uso compartilhado do sistema não implica em interferência de atribuições, sendo cada instituição responsável pelas atividades que competem à sua missão. Desta forma, as funções do sistema não são abertas aos participantes da mesma maneira, permitindo definição de níveis de acesso às informações e funções sem, no entanto, prejudicar o fluxo de informações cujo compartilhamento seja necessário.

Naturalmente, o processo de construção desta ferramenta deverá provocar reflexões diversas tanto intra quanto interinstitucionais, possivelmente acarretando a revisão de procedimentos e protocolos no âmbito da gestão integral de riscos de desastres, muitos deles com visão excessivamente centralizadora, pouco articulada e carente de refletir o verdadeiro interesse público.

\section{Objetivos do sistema}

O objetivo geral do Sistema Informatizado para Gestão Integral de Riscos de Desastres Naturais (SIGRID) é integrar as atividades dos macroprocessos para a gestão integral de riscos, melhorando o seu desempenho. Dentre os resultados, espera-se a redução de danos humanos, materiais, sociais e ambientais, o aumento da sinergia de trabalho entre os atores e a consequente otimização dos recursos públicos. O SIGRID permitirá que os gestores e tomadores de decisão tenham informações e ferramentas consistentes, além de uma visão ampla do panorama de gestão de riscos em diferentes escalas (municipal, estadual e nacional).

O sistema não visa substituir ou se tornar um modelo para a gestão organizacional de cada instituição envolvida, mas sim uma plataforma transversal, que forneça uma arquitetura dinâmica para uma gestão de riscos necessariamente interinstitucional e efetiva. Desta forma, o SIGRID não se propõe a ser uma solução padrão única e engessada à qual os atores tenham que se ajustar inflexivelmente, mas sim funcionar como peça central, ou ainda como um sistema pião (Figura 3). O SIGRID se propõe a atuar como eixo de ligação entre diferentes sistemas institucionais, acessando dados e distribuindo-os de forma dinâmica dentro de uma estrutura de hardware e software compatível, respeitando as peculiaridades e protocolos 
interinstitucionais e garantindo a interoperabilidade nas atividades de gestão integral de riscos.

O sistema também objetiva suprir os vazios operacionais existentes do ponto de vista de carência de sistemas de informação nos macroprocessos de prevenção, preparação, resposta e reconstrução, além de permitir a extração de padrões de interpretação de informações e da dinâmica de desempenho do Sistema de Proteção e Defesa Civil como um todo, nos moldes dos processos de $\mathrm{BI}$ (Business Intelligence ${ }^{7}$ ).

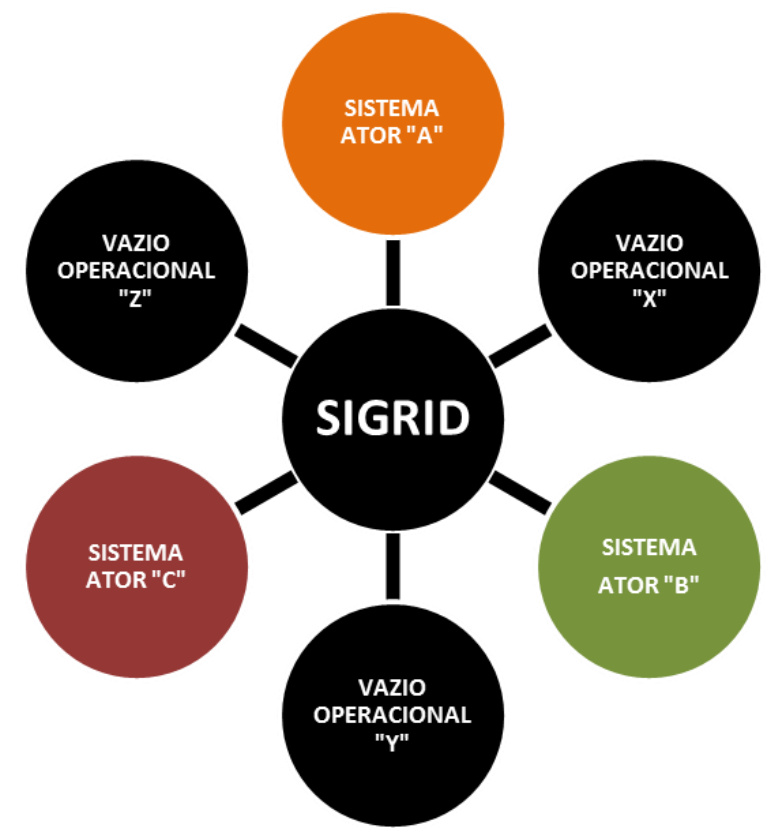

Figura 3: SIGRID como ferramenta de integração entre os atores e sistemas individuais (sistema pião). Fonte: Os autores.

Dentre os objetivos específicos do sistema, pode-se citar:

$\checkmark$ Integrar as operações e instituições relacionadas à prevenção, preparação, resposta e reconstrução;

$\checkmark$ Contribuir para padronização e implementação de protocolos interinstitucionais e atribuição clara de responsabilidades entre os atores;

$\checkmark$ Otimizar o fluxo de processos e atividades de gestão integral de riscos;

$\checkmark$ Permitir o compartilhamento dinâmico de dados e informações;

$\checkmark$ Acompanhar em tempo real as ações de prevenção, preparação, resposta e reconstrução, em diversos níveis;

\footnotetext{
7 “A terminologia Inteligência Empresarial (Business Intelligence - BI) é usada para denominar uma série de ferramentas que permitem a análise de um grande volume de dados... As ferramentas de BI são utilizadas para extrair e unificar os dados organizacionais, que muitas vezes se encontram em bases de dados diferentes, com o objetivo de transformar estes dados em informações úteis para os processos de tomadas de decisões gerenciais. Isso é possível por que essas ferramentas disponibilizam a análise de dados contextualizados, trabalham com hipóteses e relações de causas e efeitos" (ROSA, 2008).
} 
$\checkmark$ Suprir as carências principais relacionadas a sistemas de informação no âmbito da gestão integral de riscos;

$\checkmark$ Avaliar o desempenho sob diversos aspectos no âmbito da gestão integral de riscos, implementar correções e melhorias;

$\checkmark$ Otimizar as comunicações entre os atores envolvidos;

$\checkmark$ Contribuir para o desenvolvimento das culturas intra e interorganizacional baseada em melhoria contínua.

\section{Requisitos gerais do SIGRID e seus sistemas auxiliares}

Os requisitos podem ser entendidos como um conjunto de condições que o sistema deverá atender, de modo a adaptar-se ao ecossistema onde atuará e exercer suas funções com aproveitamento satisfatório. A definição dos requisitos ajuda a delimitar um perfil geral de atuação e comportamento da ferramenta.

Os requisitos gerais do SIGRID devem estar alinhados com as diretrizes da Política Nacional de Proteção e Defesa Civil - PNPDEC, podendo-se citar:

$\checkmark$ Transversalidade de utilização do sistema entre os atores envolvidos;

$\checkmark$ Integração e compartilhamento de informações de forma dinâmica, respeitadas as missões e protocolos institucionais, bem como as características do pacto federativo;

$\checkmark$ Manipulação de dados e arquivos no formato compatível com sistemas geográficos de informação (SIG);

$\checkmark$ Exportação de dados para diversos formatos, inclusive planilhas eletrônicas;

$\checkmark$ Utilização de recursos de cloud computing ${ }^{8}$, permitindo acesso remoto dos usuários;

$\checkmark$ Exercício do princípio da responsabilidade conjunta, porém diferenciada entre os atores da União, Estados, Distrito Federal e Municípios;

$\checkmark$ Abordagem sistêmica das ações de prevenção, mitigação, preparação, resposta, recuperação e reconstrução;

$\checkmark$ Valorização dos instrumentos de planejamento previstos em legislação, tais como Plano Diretor, Plano Municipal de Redução de Riscos, Plano de Contingências e Plano de Expansão Urbana;

\footnotetext{
${ }^{8} \mathrm{O}$ termo cloud computing (ou computação nas nuvens, em tradução literal) refere-se essencialmente à ideia de utilização, em qualquer lugar e independente de plataforma, as mais variadas aplicações por meio da internet com as mesmas funcionalidades de tê-las instaladas nos computadores (ALECRIM, 2008).
} 
$\checkmark$ Contribuição para a padronização das operações, monitoramento, gestão de desempenho e melhoria contínua de todo o SINPDEC;

$\checkmark$ Incorporação de inovações de forma dinâmica, colocando a tecnologia a serviço do bem estar social.

$\checkmark$ Sendo o sistema proposto de amplitude nacional, porém necessariamente com alcance local e escalas compatíveis com as áreas de risco, buscou-se realizar as análises sobre o SIGRID em conjunto com as atribuições de três recentes e importantes sistemas complementares que figuram no âmbito federal contemporâneo:

$\checkmark$ Sistema Nacional de Monitoramento e Alertas de Desastres Naturais. Este sistema já está implementado, sendo permanentemente atualizado e administrado pelo Cemaden (MCTI);

$\checkmark$ Sistema de Monitoramento da Ocupação Urbana. Este sistema foi implementado em área piloto e encontra-se em fase de estruturação do Laboratório Nacional de Computação Científica Aplicada à Análise Urbana. Trata-se de uma proposta do Departamento de Políticas de Acessibilidade e Planejamento Urbano (MCid);

$\checkmark$ Sistema Integrado de Informações Sobre Desastres (S2iD). Este sistema está sendo testado e encontra-se em fase de disseminação através de cursos de capacitação para sua utilização, sob administração da Secretaria Nacional de Defesa Civil (MI).

Os Sistemas de Monitoramento da Ocupação Urbana e o Sistema de Informações Sobre Desastres ainda não foram formalmente lançados, de modo que as análises foram realizadas sobre conteúdos de apresentações e apostilas de cursos de capacitação relacionadas a estes sistemas. Já em relação ao Sistema de Monitoramento e Alertas de Desastres Naturais as análises puderam ser feitas sobre o sistema em operação.

Como existem diversos outros sistemas informatizados em funcionamento nas mais variadas instituições que compõem o SINPDEC, especialmente no âmbito dos estados e até mesmo de alguns municípios mais preparados, recomenda-se que os três sistemas citados atuem como congregadores de informações e sistemas afins com suas respectivas missões. Ou seja, o Sistema de Monitoramento e Alertas de Desastres Naturais deve atuar como congregador de informações e sistemas existentes federais, estaduais e municipais relacionados a monitoramento e alertas de desastres (o que já acontece em relação a um grande número instituições parceiras). Da mesma forma deve atuar o Sistema de Monitoramento da 
Ocupação Urbana para fins de monitoramento e gestão da ocupação urbana, assim como o

Sistema de Informações Sobre Desastres quanto às atividades de Defesa Civil.

O aspecto congregador dos três sistemas citados é de suma relevância, pois a abrangência dos sistemas nacionais não deve conflitar com as informações de outros sistemas existentes, complementares ou concorrentes, nas esferas estadual, municipal e até mesmo federal (Figura 4).

\section{Sistemas federais, estaduais e municipais existentes de monitoramento e alertas (6) 8 (6) 6}

Dados observacionais e conhecimento provenientes de fontes diversas (4) 98

Dados e informações provenientes de sistemas complementares (via SIGRID ou não)

(i) 6

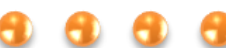

Sistema

Nacional de

Monitoramento

e Alertas de

Desastres

Naturais

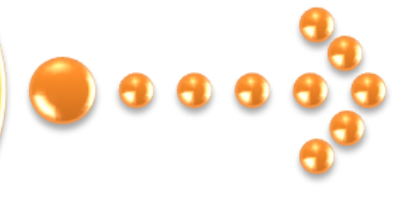

Sistemas federais, estaduais e municipais existentes de monitoramento da ocupação urbana

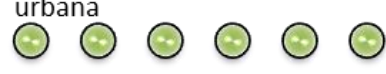

Dados observacionais e conhecimento provenientes de fontes diversas

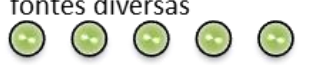

Dados e informações provenientes de sistemas complementares (via SIGRID ou não)

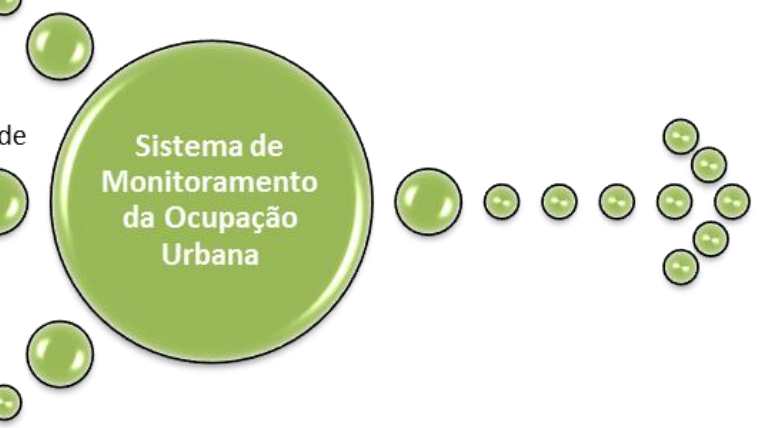

\section{SIGRID}

Sistemas federais, estaduais e municipais existentes de informações sobre desastres

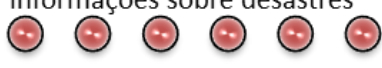

Dados observacionais e conhecimento provenientes de fontes diversas

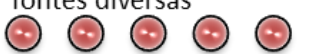

Dados e informações provenientes de sistemas complementares (via SIGRID ou não)

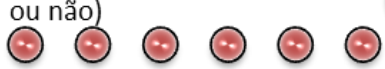

SIGRID

Figura 4: Aspecto congregador dos três principais sistemas nacionais em voga no cenário brasileiro contemporâneo de gestão integral de riscos. Fonte: Os autores. 


\section{Funcionalidades Esperadas do SIGRID e Seus Sistemas Auxiliares}

As funcionalidades de um sistema dizem respeito às tarefas que este deverá ser apto a executar. As principais funcionalidades identificadas do SIGRID e de seus sistemas auxiliares são apresentadas a seguir de acordo com os macroprocessos de PREVENÇÃO, PREPARAÇÃO, RESPOSTA e RECONSTRUÇÃO, aos quais foram associados os módulos do sistema. A coluna "Atribuição Atual" corresponde ao sistema ao qual uma determinada funcionalidade se aplica no momento. Já a coluna "Atribuição Sugerida" representa uma sugestão sobre qual sistema mostra-se mais aderente a uma determinada funcionalidade. O código ND representa que a funcionalidade em questão não é contemplada até o momento por nenhum sistema nacional (Não Disponível).

Quadro 1: Funcionalidades esperadas do MÓDULO PREVENÇÃO do SIGRID e seus sistemas auxiliares. Fonte: Os autores.

\begin{tabular}{|c|c|c|}
\hline FUNCIONALIDADES DO MÓDULO PREVENÇÃO & $\begin{array}{l}\text { ATRIBUIÇÃO } \\
\text { ATUAL }\end{array}$ & $\begin{array}{l}\text { ATRIBUIÇÃO } \\
\text { SUGERIDA }\end{array}$ \\
\hline $\begin{array}{l}\text { Realizar o monitoramento remoto / local da ocupação do solo } \\
\text { urbano e seu controle, inclusive da expansão urbana, em base } \\
\text { SIG. }\end{array}$ & $\begin{array}{l}\text { Sistema de } \\
\text { Monitoramento } \\
\text { da Ocupação } \\
\text { Urbana }\end{array}$ & A mesma. \\
\hline $\begin{array}{l}\text { Visualizar mapas de risco de desastres de forma } \\
\text { georreferenciada. }\end{array}$ & $\begin{array}{l}\text { Sistema de } \\
\text { Monitoramento e } \\
\text { Alerta de } \\
\text { Desastres } \\
\text { Naturais }\end{array}$ & A mesma. \\
\hline $\begin{array}{l}\text { Permitir o cadastro prévio e georreferenciado das residências, } \\
\text { famílias e moradores de áreas de risco, assim como de seus } \\
\text { bens em risco (casa, móveis, eletrodomésticos). }\end{array}$ & ND & $\begin{array}{l}\text { Sistema de } \\
\text { Monitoramento } \\
\text { da Ocupação } \\
\text { Urbana }\end{array}$ \\
\hline $\begin{array}{c}\text { Integração de informações com outros programas e cadastros } \\
\text { públicos federais, estaduais e municipais (Cadúnico, Bolsa } \\
\text { Família, Saúde na Família, etc.). }\end{array}$ & ND & SIGRID \\
\hline $\begin{array}{c}\text { Disponibilizar para consulta os Planos Diretores, os Planos } \\
\text { Municipais de Redução de Riscos e os Planos de Expansão } \\
\text { Urbana dos municípios, bem como outros documentos } \\
\text { normativos / legais. }\end{array}$ & Pulverizado & SIGRID \\
\hline $\begin{array}{c}\text { Realizar o acompanhamento dinâmico das ações previstas no } \\
\text { Plano Diretor, Plano Municipal de Redução de Riscos, do Plano } \\
\text { de Expansão Urbana e do Plano de Reconstrução (caso haja), } \\
\text { por parte das instâncias estaduais e federais. }\end{array}$ & ND & SIGRID \\
\hline $\begin{array}{l}\text { Agilizar a liberação de recursos aos municípios para as ações de } \\
\text { prevenção de desastres, podendo oferecer uma plataforma } \\
\text { online para elaboração dos projetos de forma padronizada, } \\
\text { permitindo interação com os órgãos fomentadores de recursos. }\end{array}$ & ND & $\begin{array}{l}\text { Sistema de } \\
\text { Monitoramento } \\
\text { da Ocupação } \\
\text { Urbana }\end{array}$ \\
\hline $\begin{array}{l}\text { Realizar registro e controle das ações de prevenção realizadas } \\
\text { sobre os indivíduos, famílias e comunidades em áreas de risco } \\
\text { (capacitações, treinamentos, notificações, realocação, aluguel } \\
\text { social, etc.). }\end{array}$ & ND & S2iD \\
\hline
\end{tabular}




\begin{tabular}{|c|c|c|}
\hline $\begin{array}{l}\text { Realizar registro e controle das ações de prevenção realizadas } \\
\text { sobre os técnicos das defesas civis, prefeituras e outros atores } \\
\text { (capacitações, treinamentos, etc.). }\end{array}$ & ND & S2iD \\
\hline $\begin{array}{c}\text { Permitir o controle de manutenção de dispositivos e } \\
\text { equipamentos da rede observacional, atuando dentro do } \\
\text { possível com a manutenção preventiva, de preferência em } \\
\text { parceria com as comunidades e prefeituras. }\end{array}$ & ND & $\begin{array}{l}\text { Sistema de } \\
\text { Monitoramento e } \\
\text { Alertas de } \\
\text { Desastres } \\
\text { Naturais }\end{array}$ \\
\hline $\begin{array}{l}\text { Permitir a visualização do histórico de dados disponíveis sobre } \\
\text { os municípios, preferencialmente com compartilhamento dos } \\
\text { dados do IBGE. }\end{array}$ & IBGE & $\begin{array}{l}\text { Sistema de } \\
\text { Monitoramento } \\
\text { da Ocupação } \\
\text { Urbana }\end{array}$ \\
\hline $\begin{array}{l}\text { Permitir a visualização de dados sobre o histórico de desastres e } \\
\text { registro de ocorrências da defesa civil de cada município. }\end{array}$ & $\begin{array}{l}\text { Sistema de } \\
\text { Informações } \\
\text { Sobre Desastres }\end{array}$ & A mesma. \\
\hline $\begin{array}{l}\text { Disponibilizar e visualizar mapas e cartas diversos dos } \\
\text { municípios, preferencialmente nos formatos compatíveis com } \\
\text { sistemas de informações geográficas e também em PDF (mapas } \\
\text { políticos, cartas geológico-geotécnicas, mapas de uso e } \\
\text { ocupação do solo, etc.). }\end{array}$ & $\begin{array}{l}\text { Sistema de } \\
\text { Monitoramento } \\
\text { da Ocupação } \\
\text { Urbana }\end{array}$ & A mesma. \\
\hline $\begin{array}{l}\text { Produzir camadas temáticas que mostrem padrões espectrais } \\
\text { de urbanização, com base em dados de satélite multiespectrais } \\
\text { livres e programas de código aberto. }\end{array}$ & $\begin{array}{l}\text { Sistema de } \\
\text { Monitoramento } \\
\text { da Ocupação } \\
\text { Urbana }\end{array}$ & A mesma. \\
\hline $\begin{array}{l}\text { Permitir visualização e atualização do efetivo das defesas civis } \\
\text { municipais, sua rotatividade, instalações e equipamentos. }\end{array}$ & ND & S2iD \\
\hline $\begin{array}{l}\text { Permitir a padronização dos procedimentos e documentos } \\
\text { relacionados às atividades de prevenção, tanto intra quanto } \\
\text { interinstitucionais. }\end{array}$ & Pulverizado & SIGRID \\
\hline $\begin{array}{l}\text { Realizar intercâmbio de dados e informações passíveis de } \\
\text { compartilhamento, entre os diferentes sistemas existentes. }\end{array}$ & ND & SIGRID \\
\hline $\begin{array}{l}\text { Permitir o desenvolvimento e utilização de métricas de } \\
\text { desempenho das atividades de prevenção, por meio de } \\
\text { indicadores de desempenho e definições de padrões e metas. }\end{array}$ & ND & SIGRID \\
\hline $\begin{array}{l}\text { Gerar relatórios visando retratar aspectos diversos das } \\
\text { atividades de prevenção realizadas e seu desempenho. }\end{array}$ & ND & SIGRID \\
\hline
\end{tabular}

Quadro 2: Funcionalidades esperadas do MÓDULO PREPARAÇÃO do SIGRID e seus sistemas auxiliares. Fonte: Os autores.

\begin{tabular}{|c|c|c|}
\hline FUNCIONALIDADES DO MÓDULO PREPARAÇÃO & $\begin{array}{l}\text { ATRIBUIÇÃO } \\
\text { ATUAL }\end{array}$ & $\begin{array}{l}\text { ATRIBUIÇÃO } \\
\text { SUGERIDA }\end{array}$ \\
\hline $\begin{array}{l}\text { Realizar monitoramento meteorológico, monitoramento } \\
\text { de processos hidrológico e de processos geodinâmicos, } \\
\text { visando à previsão de desastres naturais. }\end{array}$ & $\begin{array}{c}\text { Sistema de } \\
\text { Monitoramento } \\
\text { e Alerta de } \\
\text { Desastres } \\
\text { Naturais }\end{array}$ & A mesma. \\
\hline $\begin{array}{c}\text { Realizar modelagens de processos hidrológicos e } \\
\text { geodinâmicos, visando à previsão de desastres naturais. }\end{array}$ & $\begin{array}{c}\text { Sistema de } \\
\text { Monitoramento } \\
\text { e Alerta de } \\
\text { Desastres } \\
\text { Naturais }\end{array}$ & A mesma. \\
\hline $\begin{array}{c}\text { Realizar análises e emitir alertas de desastres naturais para } \\
\text { o Centro Nacional de Gerenciamento de Riscos de } \\
\text { Desastres (CENAD). }\end{array}$ & $\begin{array}{c}\text { Sistema de } \\
\text { Monitoramento } \\
\text { e Alerta de } \\
\text { Desastres } \\
\text { Naturais }\end{array}$ & A mesma. \\
\hline
\end{tabular}




\begin{tabular}{|c|c|c|}
\hline $\begin{array}{c}\text { Agilizar a liberação de recursos para as ações de } \\
\text { preparação, podendo oferecer uma plataforma online para } \\
\text { elaboração dos projetos de forma padronizada, permitindo } \\
\text { interação com os órgãos fomentadores de recursos } \\
\text { (Ministério das Cidades e Ministério da Integração). }\end{array}$ & Pulverizado & S2iD \\
\hline $\begin{array}{c}\text { Registrar e controlar das ações de preparação realizadas } \\
\text { sobre os indivíduos, famílias e comunidades (treinamentos, } \\
\text { simulados, etc.). }\end{array}$ & ND & S2iD \\
\hline $\begin{array}{c}\text { Registrar e controlar as ações de preparação realizadas } \\
\text { sobre os técnicos de defesa civil, prefeituras e outros } \\
\text { atores (treinamentos, simulados, capacitações sobre } \\
\text { alertas e seu significado, etc.). }\end{array}$ & ND & S2iD \\
\hline $\begin{array}{c}\text { Visualizar a estrutura de aparelhamento e apoio logístico } \\
\text { disponível nos municípios. }\end{array}$ & ND & S2iD \\
\hline $\begin{array}{c}\text { Disponibilizar de forma organizada as informações do } \\
\text { Plano de Contingências para todos os envolvidos, bem } \\
\text { como de outros instrumentos normativos / legais. }\end{array}$ & ND & S2iD \\
\hline $\begin{array}{c}\text { Permitir a padronização dos procedimentos e documentos } \\
\text { relacionados às atividades de preparação, tanto intra } \\
\text { quanto interinstitucionais. }\end{array}$ & Pulverizado & SIGRID \\
\hline $\begin{array}{c}\text { Realizar intercâmbio de dados e informações passíveis de } \\
\text { compartilhamento, entre os diferentes sistemas existentes. }\end{array}$ & ND & SIGRID \\
\hline $\begin{array}{c}\text { Permitir o desenvolvimento e utilização de métricas de } \\
\text { desempenho das atividades de preparação, por meio de } \\
\text { indicadores de desempenho e definições de padrões e } \\
\text { metas. }\end{array}$ & ND & SIGRID \\
\hline $\begin{array}{c}\text { Gerar relatórios visando retratar aspectos diversos das } \\
\text { atividades de preparação realizadas e seu desempenho. }\end{array}$ & ND & \\
\hline
\end{tabular}

Quadro 3: Funcionalidades esperadas do MÓDULO RESPOSTA do SIGRID e seus sistemas auxiliares. Fonte: Os autores.

\begin{tabular}{|c|c|c|}
\hline FUNCIONALIDADES DO MÓDULO RESPOSTA & $\begin{array}{l}\text { ATRIBUIÇÃO } \\
\text { ATUAL }\end{array}$ & $\begin{array}{l}\text { ATRIBUIÇÃO } \\
\text { SUGERIDA }\end{array}$ \\
\hline $\begin{array}{l}\text { Realizar o cruzamento de informações sobre os atingidos por } \\
\text { desastres e atualização de listas de desabrigados, desalojados, } \\
\text { hospitalizados, desaparecidos, óbitos, etc., podendo haver site } \\
\text { com a disponibilização destas informações. }\end{array}$ & ND & S2iD \\
\hline $\begin{array}{l}\text { Contribuir para a agilidade e precisão das avaliações de danos } \\
\text { mediante interligação com os cadastros da fase de prevenção. }\end{array}$ & ND & S2iD \\
\hline $\begin{array}{c}\text { Realizar rápida identificação dos atingidos através de cruzamentos } \\
\text { com os cadastros da fase de prevenção, com verificações anti- } \\
\text { fraude. }\end{array}$ & ND & S2iD \\
\hline $\begin{array}{l}\text { Contribuir para o acompanhamento dinâmico das ações de } \\
\text { resposta, por parte das instâncias estaduais e federais. }\end{array}$ & ND & S2iD \\
\hline $\begin{array}{l}\text { Disponibilização de legislação e instrumentos burocráticos } \\
\text { relacionados a contratações em situações de emergência. }\end{array}$ & S2iD & A mesma \\
\hline $\begin{array}{l}\text { Agilizar a liberação de recursos para as ações de resposta, } \\
\text { podendo oferecer uma plataforma online para elaboração dos } \\
\text { pedidos de recurso de forma padronizada, permitindo interação }\end{array}$ & S2iD & A mesma \\
\hline
\end{tabular}


PROPOSTA PARA A CONSTRUÇÃO UM SISTEMA INFORMATIZADO PARA GESTÃO INTEGRAL DE RISCOS DE DESASTRES NATURAIS (SIGRID) NO CENÁRIO BRASILEIRO Leandro Torres Di Gregorio, Carlos Alberto Pereira Soares, Silvia Midori Saito, Erico Soriano, Luciana de Resende Londe, Marcos Pellegrini Coutinho

\begin{tabular}{|c|c|c|}
\hline $\begin{array}{l}\text { com os órgãos fomentadores de recursos (Ministério da } \\
\text { Integração). }\end{array}$ & & \\
\hline $\begin{array}{l}\text { Contribuir para o registro e controle das ações de resposta } \\
\text { realizadas sobre os indivíduos, famílias e comunidades (abrigo, } \\
\text { aluguel social, doações, atendimentos médicos e psicológicos, } \\
\text { concessão de benefícios sociais, etc.). }\end{array}$ & ND & S2iD \\
\hline $\begin{array}{l}\text { Contribuir para o acompanhamento da evolução da situação dos } \\
\text { atingidos no tempo, até o momento da provisão habitacional } \\
\text { definitiva, permitindo adequar as ações necessárias para o grupo } \\
\text { afetado. }\end{array}$ & ND & S2iD \\
\hline $\begin{array}{c}\text { Fornecer ferramentas práticas que auxiliem o Administrador do } \\
\text { Desastre a planejar, executar e controlar as atividades de socorro } \\
\text { às populações em risco. }\end{array}$ & ND & S2iD \\
\hline $\begin{array}{l}\text { Fornecer ferramentas práticas que auxiliem o Administrador do } \\
\text { Desastre a planejar, executar e controlar as atividades de } \\
\text { assistência às populações afetadas (logísticas, assistenciais e de } \\
\text { promoção da saúde). }\end{array}$ & ND & S2iD \\
\hline $\begin{array}{l}\text { Fornecer ferramentas práticas que auxiliem o Administrador do } \\
\text { Desastre a planejar, executar e controlar as atividades de } \\
\text { reabilitação dos cenários dos desastres (avaliação de danos, } \\
\text { vistoria e elaboração de laudos técnicos, desmontagem de } \\
\text { estruturas danificadas, desobstrução e remoção de escombros, } \\
\text { sepultamento, limpeza, descontaminação, desinfecção e } \\
\text { desinfestação do ambiente, reabilitação dos serviços essenciais, } \\
\text { recuperação de unidades habitacionais de baixa renda). }\end{array}$ & ND & S2iD \\
\hline $\begin{array}{l}\text { Contribuir para o registro e controle das ações de resposta } \\
\text { realizadas pelos atores envolvidos na fase de resposta, inclusive } \\
\text { organizações não governamentais e voluntários, inclusive com } \\
\text { acesso a um "diário de operações". }\end{array}$ & ND & S2iD \\
\hline $\begin{array}{l}\text { Disponibilizar de forma organizada as informações do Plano de } \\
\text { Contingências para todos os envolvidos. }\end{array}$ & ND & S2iD \\
\hline $\begin{array}{l}\text { Permitir o registro georreferenciado e caracterização padronizada } \\
\text { dos desastres, permitindo aprimoramento do sistema de } \\
\text { monitoramento e alerta. }\end{array}$ & S2iD & A mesma. \\
\hline $\begin{array}{c}\text { Permitir a atualização dinâmica de um banco de dados sobre } \\
\text { desastres naturais. }\end{array}$ & S2iD & A mesma. \\
\hline $\begin{array}{l}\text { Permitir o lançamento de despesas e a gestão de custos } \\
\text { transparente em situações de resposta. }\end{array}$ & ND & S2iD \\
\hline $\begin{array}{l}\text { Permitir a padronização dos procedimentos e documentos } \\
\text { relacionados às atividades de resposta, tanto intra quanto } \\
\text { interinstitucionais, baseados especialmente na metodologia } \\
\text { SCO/ICS. }\end{array}$ & Pulverizado & SIGRID \\
\hline $\begin{array}{l}\text { Realizar intercâmbio de dados e informações passíveis de } \\
\text { compartilhamento, entre os diferentes sistemas existentes. }\end{array}$ & ND & SIGRID \\
\hline $\begin{array}{l}\text { Permitir o desenvolvimento e utilização de métricas de } \\
\text { desempenho das atividades de resposta, por meio de indicadores } \\
\text { de desempenho e definições de padrões e metas. }\end{array}$ & ND & SIGRID \\
\hline $\begin{array}{l}\text { Gerar relatórios visando retratar aspectos diversos das atividades } \\
\text { de resposta realizadas e seu desempenho. }\end{array}$ & ND & SIGRID \\
\hline
\end{tabular}

Quadro 4: Funcionalidades esperadas do MÓDULO RECONSTRUÇÃO do SIGRID e seus sistemas auxiliares. Fonte: Os autores.

\begin{tabular}{|l|l|l} 
FUNCIONALIDADES DO MÓDULO RECONSTRUÇÃO & ATRIBUIÇÃO & ATRIBUIÇÃO
\end{tabular}


PROPOSTA PARA A CONSTRUÇÃO UM SISTEMA INFORMATIZADO PARA GESTÃO INTEGRAL DE RISCOS DE DESASTRES NATURAIS (SIGRID) NO CENÁRIO BRASILEIRO Leandro Torres Di Gregorio, Carlos Alberto Pereira Soares, Silvia Midori Saito, Erico Soriano, Luciana de Resende Londe, Marcos Pellegrini Coutinho

\begin{tabular}{|c|c|c|}
\hline & ATUAL & SUGERIDA \\
\hline $\begin{array}{l}\text { Agilizar a liberação de recursos para as ações de reconstrução, } \\
\text { podendo oferecer uma plataforma online para elaboração dos } \\
\text { projetos de forma padronizada, permitindo interação com os } \\
\text { órgãos fomentadores de recursos (Ministério das Cidades). }\end{array}$ & Pulverizado & $\begin{array}{l}\text { Sistema de } \\
\text { Monitoramento } \\
\text { da Ocupação } \\
\text { Urbana }\end{array}$ \\
\hline $\begin{array}{l}\text { Contribuir para o registro e controle das ações de reconstrução } \\
\text { realizadas sobre os indivíduos, famílias e comunidades (provisão } \\
\text { habitacional, indenizações, aluguel social, etc.). }\end{array}$ & ND & $\begin{array}{l}\text { Sistema de } \\
\text { Monitoramento } \\
\text { da Ocupação } \\
\text { Urbana }\end{array}$ \\
\hline $\begin{array}{l}\text { Disponibilizar de forma organizada as informações do Plano de } \\
\text { Reconstrução (habitação, infraestrutura, aspectos } \\
\text { socioeconômicos e psicossociais) para todos os envolvidos. }\end{array}$ & ND & $\begin{array}{l}\text { Sistema de } \\
\text { Monitoramento } \\
\text { da Ocupação } \\
\text { Urbana }\end{array}$ \\
\hline $\begin{array}{c}\text { Disponibilização de instrumentos normativos e legais relacionados } \\
\text { à reconstrução. }\end{array}$ & Pulverizado & $\begin{array}{l}\text { Sistema de } \\
\text { Monitoramento } \\
\text { da Ocupação } \\
\text { Urbana }\end{array}$ \\
\hline $\begin{array}{l}\text { Permitir o acompanhamento dinâmico das ações do Plano de } \\
\text { Reconstrução por parte das instâncias estaduais e federal } \\
\text { (progresso das obras e custos, especialmente), de forma } \\
\text { georreferenciada. }\end{array}$ & ND & $\begin{array}{l}\text { Sistema de } \\
\text { Monitoramento } \\
\text { da Ocupação } \\
\text { Urbana }\end{array}$ \\
\hline $\begin{array}{l}\text { Viabilizar o monitoramento da ocupação do estoque de terrenos } \\
\text { disponíveis para provisão habitacional de interesse social. }\end{array}$ & ND & $\begin{array}{l}\text { Sistema de } \\
\text { Monitoramento } \\
\text { da Ocupação } \\
\text { Urbana }\end{array}$ \\
\hline $\begin{array}{l}\text { Permitir o acompanhamento da recuperação econômica do } \\
\text { município. }\end{array}$ & ND & $\begin{array}{l}\text { Sistema de } \\
\text { Monitoramento } \\
\text { da Ocupação } \\
\text { Urbana }\end{array}$ \\
\hline $\begin{array}{l}\text { Visualização das informações sobre o histórico das ações de } \\
\text { reconstrução. }\end{array}$ & ND & $\begin{array}{l}\text { Sistema de } \\
\text { Monitoramento } \\
\text { da Ocupação } \\
\text { Urbana }\end{array}$ \\
\hline $\begin{array}{l}\text { Permitir a padronização dos procedimentos e documentos } \\
\text { relacionados às atividades de reconstrução, tanto intra quanto } \\
\text { interinstitucionais. }\end{array}$ & Pulverizado & SIGRID \\
\hline $\begin{array}{l}\text { Realizar intercâmbio de dados e informações passíveis de } \\
\text { compartilhamento, entre os diferentes sistemas existentes. }\end{array}$ & ND & SIGRID \\
\hline $\begin{array}{c}\text { Permitir o desenvolvimento e utilização de métricas de } \\
\text { desempenho das atividades de reconstrução, por meio de } \\
\text { indicadores de desempenho e definições de padrões e metas. }\end{array}$ & ND & SIGRID \\
\hline $\begin{array}{l}\text { Gerar relatórios visando retratar aspectos diversos das atividades } \\
\text { de reconstrução realizadas e seu desempenho. }\end{array}$ & ND & SIGRID \\
\hline
\end{tabular}

\section{CONCLUSÃO}

No atual cenário brasileiro, a gestão integral de riscos de desastres representa um desafio às instituições que compõem o Sistema Nacional de Proteção e Defesa Civil. Integração de atividades e processos, uniformização de procedimentos e protocolos, melhoria do fluxo de informações e comunicações entre as instituições, são alguns dos pontos que necessitam de premente reformulação. 
Para fazer frente a estas necessidades, propôs-se a criação de um Sistema Informatizado para Gestão Integral de Riscos de Desastres Naturais (SIGRID).

Caso implementado, o SIGRID terá as funções principais de integrar os três principais sistemas nacionais (atuando como um sistema pião), suprir vazios operacionais que não sejam abordados pelos três sistemas e atuar como instância de medição e avaliação de desempenho de todas as operações que compõem direta ou indiretamente o SINPDEC. Desta forma, o SIGRID fornecerá ferramentas decisórias importantes aos gestores (nos moldes dos processos de business intelligence) e contribuirá para a consolidação da cultura de melhoria contínua em todo o SINPDEC.

Entretanto, é importante ressaltar que a construção e implementação de um sistema informatizado não são por si mesmas suficientes para garantir a efetividade das operações sistêmicas da vida real. O sistema informatizado deve ser entendido como uma ferramenta poderosa que visa auxiliar a operação e gestão do sistema real, contribuindo para integração e padronização de operações, facilitando as comunicações dos envolvidos, armazenando e organizando dados e informações de forma eficaz, bem como permitindo análises e decisões gerenciais sobre o conjunto.

Para que um sistema informatizado venha a exercer todo o potencial que possui, torna-se indispensável o trabalho de gestão direcionado aos diversos usuários, ressaltando-se a importância da liderança, treinamentos e capacitações na operação do sistema, análise crítica sobre os resultados e, sobretudo uma cultura de comprometimento dos envolvidos com a melhoria contínua. A busca da melhoria contínua na gestão de riscos é enfatizada pela NBR 31000 através do estabelecimento de metas de desempenho organizacional (no caso, multiorganizacional), da mensuração e de análises críticas, além das subsequentes mudanças de processos, sistemas, recursos, capacidade e habilidades.

Além de tudo, a clara definição de responsabilidades e a figura dos coordenadores do sistema em nível federal, estadual e municipal são entendidas como fundamentais para o sucesso do projeto, uma vez que o sistema perpassa todos os entes federativos.

Ressalta-se ainda a necessidade de avaliação e atualização periódica do próprio sistema, de forma a incorporar de forma contínua as melhores práticas organizacionais e outras inovações relacionadas ao tema. 


\section{REFERÊNCIAS BIBLIOGRÁFICAS}

ALECRIM, E. O que é Cloud Computing (Computação nas Nuvens)? Algumas características da Cloud Computing. Infowester, São Paulo, 2008. Disponível em:<http://www.infowester.com/cloudcomputing.php>. Acesso em: $18 \mathrm{dez} .2012$.

ALMEIDA, L. Q. de; PASCOALINO, A. Gestão de Risco, Desenvolvimento e (meio) Ambiente no Brasil - Um estudo de caso sobre os desastres naturais em Santa Catarina. In: SIMPÓSIO BRASILEIRO DE GEOGRAFIA FÍSICA APLICADA, 13, 2009. Viçosa (MG). Anais... XIII SBG-FA, 2009.

Disponível em: <http://www.geo.ufv.br/simposio/simposio/trabalhos/trabalhos_completos/eixo11/061.pdf > Acesso em: 12 jul. 2012.

ASSOCIAÇÃO BRASILEIRA DE NORMAS TÉCNICAS. NBR ISO 9001. Sistemas de Gestão da Qualidade - requisitos. Rio de Janeiro: ABNT, 2000, 30p.

ASSOCIAÇÃO BRASILEIRA DE NORMAS TÉCNICAS: NBR ISO 31000. Gestão de Riscos Princípios e Diretrizes. Rio de Janeiro: ABNT, 2009, 24p.

BRASIL. Lei no 12.608, de 10 de abril de 2012. Institui a Política Nacional de Proteção e Defesa Civil - PNPDEC; dispõe sobre o Sistema Nacional de Proteção e Defesa Civil - SINPDEC e o Conselho Nacional de Proteção e Defesa Civil - CONPDEC. Presidência da República. Casa Civil. Subchefia para Assuntos Jurídicos. Disponível em: <http://www.planalto.gov.br/ccivil 03/ Ato2011-2014/2012/Lei/L12608.htm> Acesso em: 15 set. 2012.

CASTRO, A.L.C. Manual de Planejamento em Defesa Civil. Brasília: Ministério da Integração Nacional (MI),1999. 133p.

MARCELINO, E. Desastres Naturais e Geotecnologias: conceitos básicos. Caderno Didático 1. Santa Maria: INPE/CRS, 2008. 40p.

ROSA, C M. O Uso de Ferramenta de Business Intelligence na Gestão Pública da Prefeitura Municipal de Passo Fundo. In: I MOSTRA DE PESQUISA DE PÓS-GRADUAÇÃO DA FACULDADE MERIDIONAL - IMED. 2008, Passo Fundo (RS). Anais... I IMED, 2008. Disponível em: 
<http://mic.imed.edu.br/anais/edicoes_anteriores/2008/Artigos/Cristiane\%20M\%20da\%20

Rosa.pdf>. Acesso em: 18 dez. 2012.

VALENCIO, N.; VALENCIO, A. Os desastres como indícios da vulnerabilidade do Sistema Nacional de Defesa Civil: o caso brasileiro. Territorium, Coimbra, v.18, p.147-156, 2011.

VARGAS. H. R .A. Guía Municipal para la Gestión del Riesgo. 2010. Disponível em: <http://www.sigpad.gov.co/sigpad/archivos/GMGRColombia.pdf>. Acesso em: 18 dez. 2012.

Artigo recebido em 20/12/2012.

Artigo aceito em 31/07/2013. 